\title{
A note on two conjectures
}

\author{
by \\ JIAHAI KAN (Nanjing)
}

1. Introduction. Let $d(n)$ denote the divisor function, $\nu(n)$ the number of distinct prime factors, and $\Omega(n)$ the total number of prime factors of $n$, respectively. About 50 years ago P. Erdös formulated the following conjectures.

(C) There exist infinitely many positive integers $n$ for which

$$
d(n)=d(n+1) .
$$

(D) There exist infinitely many positive integers $n$ for which

$$
\Omega(n)=\Omega(n+1) .
$$

(E) There exist infinitely many positive integers $n$ for which

$$
\nu(n)=\nu(n+1) .
$$

These conjectures have been studied by many mathematicians, e.g. [1], [2], [4], [6] and [8]. Although (C) and (D) were proved by Heath-Brown (cf. [4]) in 1984, conjecture (E) remains open (cf. [8]).

In 1927 during a conversation with H. Hasse, E. Artin enunciated the following famous hypothesis, now known as Artin's conjecture.

(A) For any given non-zero integer $a$ other than $1,-1$, or a perfect square, there exist infinitely many primes $p$ for which $a$ is a primitive root $(\bmod p)$.

This conjecture is the focal point of diverse areas of mathematics such as group theory, algebraic and analytic number theory, and algebraic geometry (cf. [10]). There is a vast amount of literature for conjecture (A), e.g. [7], [3] and $[5]$.

The purpose of this paper is to show the following.

TheOrem. At least one of the two conjectures (A) and (E) is true.

2000 Mathematics Subject Classification: 11B, 11N, 11P. 
2. The Lemma. Throughout the paper $p$ and $q$ denote primes.

Lemma. For sufficiently large $x$ and any fixed positive integers $K, M$ with $(K, M)=1,(2 K+1, M)=1$, we have

$$
\begin{array}{r}
\#\left\{q: q<x, 2 q+1=p \text { or } p_{1} p_{2}, p_{1}<p_{2}, q \equiv K(\bmod M)\right\} \\
\quad>\frac{C}{\varphi(M)} x \ln ^{-2} x
\end{array}
$$

where $\varphi$ denotes the Euler totient function.

Proof. This is an easy generalization of [9, Lemma 1], with $a=2, b=1$.

3. Proof of the Theorem. From the Lemma it is easy to see that at least one of the following two cases must hold.

(i) For sufficiently large $x$ and some positive integers $K, M$ with $(K, M)$ $=1,(2 K+1, M)=1$,

$$
\#\left\{q: q<x, 2 q+1=p_{1} p_{2}, p_{1}<p_{2}, q \equiv K(\bmod M)\right\} \gg x \ln ^{-2} x .
$$

(ii) For sufficiently large $x$ and any fixed positive integers $K, M$ with $(K, M)=1,(2 K+1, M)=1$,

$$
\#\{q: q<x, 2 q+1=p, q \equiv K(\bmod M)\}>\frac{C}{\varphi(M)} x \ln ^{-2} x .
$$

Let $n=2 q, n+1=p_{1} p_{2}$ in (i). It is easy to see that (i) implies conjecture (E) with $\nu(n)=\nu(n+1)=2$, and moreover, all these $n$ belong to a given arithmetic progression. We proceed to show that (ii) implies conjecture (A).

Let $a$ denote a given non-zero integer other than $1,-1$, or a perfect square. From (ii) there are infinitely many pairs of primes $p, q$ with $p-1=2 q$ and $(p, a)=1$.

By Fermat's little theorem $a^{p-1} \equiv 1(\bmod p)$ and $p-1=2 q$, we have

$$
a^{2 q} \equiv 1(\bmod p) .
$$

Consider the following two possibilities.

Case 1: $a^{2} \equiv 1(\bmod p)$. For sufficiently large $p$ this is impossible.

Case 2: $a^{q} \equiv 1(\bmod p)$. We show this is also impossible for suitably chosen $p$ and $q$.

Since $a$ is not a perfect square there is a residue class $b(\bmod 4|a|)$, with $b$ coprime to $4|a|$, such that $a$ is a quadratic non-residue of $p$ whenever $p$ is congruent to $b$ modulo $4|a|$. If we choose $M=2|a|$ and $K=(b-1) / 2$ we then see that Case 2 will not arise.

Therefore $a$ must be a primitive root $(\bmod p)$, and (ii) implies conjecture $(\mathrm{A})$.

The proof of the Theorem is complete. 


\section{References}

[1] P. Erdös and L. Mirsky, The distribution of the values of the divisor function $d(n)$, Proc. London Math. Soc. (3) 2 (1952), 257-271.

[2] P. Erdös, C. Pomerance and A. Sárközy, On locally repeated values of certain arithmetic functions II, Acta Math. Hungar. 49 (1987), 251-259.

[3] R. Gupta and M. R. Murty, A remark on Artin's conjecture, Invent. Math. 78 (1984), 127-130.

[4] D. R. Heath-Brown, The divisor function at consecutive integers, Mathematika 31 (1984), 141-149.

[5] - Artin's conjecture for primitive roots, Quart. J. Math. Oxford (2) 37 (1986), $27-38$.

[6] A. Hildebrand, The divisor function at consecutive integers, Pacific J. Math. 129 (1987), 307-319.

[7] C. Hooley, On Artin's conjecture, J. Reine Angew. Math. 226 (1967), 209-220.

[8] J. H. Kan, On the number-theoretic functions $\nu(n)$ and $\Omega(n)$, Acta Arith. 78 (1996), 91-97.

[9] J. H. Kan and Z. Shan, On the divisor function d(n), Mathematika 43 (1996), $320-322$.

[10] M. R. Murty, Artin's conjecture for primitive roots, Math. Intelligencer 10 (1988), no. $4,59-67$.

Nanjing Institute of Post \& Telecommunication

210003 Nanjing, Nanjing, China

Received on 30.4.2001

and in revised form on 14.9.2001 